ARTICLE

\title{
Artificial van der Waals hybrid synapse and its application to acoustic pattern recognition
}

Seunghwan Seo ${ }^{1}$, Beom-Seok Kang ${ }^{1}$, Je-Jun Lee ${ }^{1}$, Hyo-Jun Ryu ${ }^{1}{ }^{1}$, Sungjun Kim ${ }^{1,3}$, Hyeongjun Kim¹, Seyong Oh , Jaewoo Shim (i) ${ }^{4}$, Keun Heo ${ }^{1}$, Saeroonter Oh (i) ${ }^{5}$ \& Jin-Hong Park (i) ${ }^{1,6 凶}$

Brain-inspired parallel computing, which is typically performed using a hardware neuralnetwork platform consisting of numerous artificial synapses, is a promising technology for effectively handling large amounts of informational data. However, the reported nonlinear and asymmetric conductance-update characteristics of artificial synapses prevent a hardware neural-network from delivering the same high-level training and inference accuracies as those delivered by a software neural-network. Here, we developed an artificial van-der-Waals hybrid synapse that features linear and symmetric conductance-update characteristics. Tungsten diselenide and molybdenum disulfide channels were used selectively to potentiate and depress conductance. Subsequently, via training and inference simulation, we demonstrated the feasibility of our hybrid synapse toward a hardware neural-network and also delivered high recognition rates that were comparable to those delivered using a software neural-network. This simulation involving the use of acoustic patterns was performed with a neural network that was theoretically formed with the characteristics of the hybrid synapses.

\footnotetext{
${ }^{1}$ Department of Electrical and Computer Engineering, Sungkyunkwan University, Suwon 16419, Korea. ${ }^{2}$ Semiconductor R\&D Center, Samsung Electronics Co. Ltd, Hwasung 18448, Korea. ${ }^{3}$ Foundry Division, Samsung Electronics Co. Ltd., Youngin 17113, Korea. ${ }^{4}$ Department of Mechanical Engineering,

Massachusetts Institute of Technology (MIT), Cambridge, MA 02139, USA. ${ }^{5}$ Division of Electrical Engineering, Hanyang University, Ansan 15588, Korea.

${ }^{6}$ Sungkyunkwan Advanced Institute of Nanotechnology (SAINT), Sungkyunkwan University, Suwon 16417, Korea. ${ }^{凶}$ email: jhpark9@skku.edu
} 
$t$ is predicted that a large amount of unstructured data in the upcoming Big Data era will not be processed efficiently via conventional serial computing technology based on the von Neumann architecture ${ }^{1}$. Thus, a brain-inspired parallel computing technology suitable for dealing with such unstructured data was recently proposed ${ }^{2,3}$. Brain-inspired computing is generally performed using a hardware neural-network (HW-NN) platform consisting of numerous artificial synapses ${ }^{4,5}$. Therefore, considerable effort has been directed toward the implementation of artificial synapses mimicking the behavior of biological synapses, such as short-term plasticity and long-term plasticity ${ }^{6,7}$. Synaptic devices based on various operating mechanisms and materials have been reported, including resistive random-access memory (RRAM), phase-change memory (PCM), field-effect transistors (FETs) with ferroelectric or charge-trapping layer, electrochemical memory, optoelectronic memory ${ }^{8-20}$. However, it has not been demonstrated that an HW-NN composed of such synaptic devices can perform training and inference tasks with the same level of accuracy as a software-based neural-network (SW-NN). This is because such devices do not sufficiently satisfy the synaptic characteristics, such as the cycle-to-cycle variation $(\mathrm{CCV})$, device-to-device variation (DDV), retention time, endurance, number of conductance states, dynamic range, and linear/symmetrical conductance change $\mathrm{e}^{20-23}$. In particular, the linearity and symmetricity of the conductance change are known to significantly affect the inference accuracy after the training process of $\mathrm{HW}-\mathrm{NNs}^{24,25}$.

J. Woo et al. and S. Park et al. reported $\mathrm{HfO}_{\mathrm{x}}$ RRAM and $\mathrm{AlO}_{\mathrm{x}} /$ TiN PCMO-based synaptic devices, respectively, where the nonlinear and asymmetrical conductance change resulted in a low inference accuracy of $<40 \%$ for the Modified National Institute of Standards and Technology (MNIST) dataset ${ }^{26,27}$. For the $\mathrm{HfO}_{\mathrm{x}}$ RRAM, an $\mathrm{AlO}_{\mathrm{x}}$ barrier layer was introduced, consequently improving its conductance change more linearly, but this device inherently presented low dynamic range and high $\mathrm{CCV}^{18,26}$. For the hafnium-zirconium oxide FeFET-type synapse reported by $\mathrm{M}$. Jerry et al., a highly symmetric conductance change was achieved, leading to a high inference accuracy of $90 \%$ for the MNIST dataset $^{28}$. However, in the FeFET synapse, non-identical spikes were applied for controlling the conductance state. This is because most of the reported synaptic devices operate on the basis of a physical mechanism that cannot change the conductance linearly with respect to the applied voltage. Meanwhile, E. J. Fuller et al. reported an ionic floating gate (IFG)-based synaptic device featuring a very linear conductance change that was achieved by a gradual composition modulation in the $\mathrm{IFG}^{29}$. Recently, various studies to approach the training/inference accuracy of an SW-NN have been attempted by designing synaptic unit cells with highly linear and symmetric conductance change characteristic ${ }^{24,30,31}$. $\mathrm{S}$. Kim et al. reported a very linear conductance change in their synaptic unit cell consisting of three transistors and one capacitor $^{30}$. Although the excellent linearity allowed an accurate training process, the volatility of the cell made inference based on the trained information difficult. S. Ambrogio et al. and X. Sun et al. applied nonvolatile memory elements, such as PCM and ferroelectric capacitors, to their synapse cells, yielding high training and inference accuracies simultaneously ${ }^{24,31}$. However, such synapse cells require highly complex peripheral circuits for operation, as well as a large number of devices. Therefore, additional studies on artificial synapses should be performed to achieve desirable synaptic characteristics required for highperformance HW-NNs.

Herein, we report an artificial vdW-hybrid synaptic device that features linear and symmetric conductance change characteristics. The excellent conductance controllability is accomplished by using tungsten diselenide $\left(\mathrm{WSe}_{2}\right)$ and molybdenum disulfide
$\left(\mathrm{MoS}_{2}\right)$ hybrid channels, which are specialized for linear conductance potentiation and depression, respectively. We also discuss the $\mathrm{CCV} \& \mathrm{DDV}$, relative standard deviation (RSD), endurance, symmetricity, and dynamic range for the long-term potentiation (LTP)/long-term depression (LTD) characteristic curves with respect to the conditions of weight control spikes. In particular, our synaptic device is investigated and compared with other devices reported heretofore, in terms of various synaptic characteristics mentioned above, weight updating energy, and active area (see Supplementary Table 1). Finally, we demonstrate the feasibility of the vdW-hybrid synaptic device for an HW-NN and present high recognition rates close to those for an SW-NN via training and inference simulation, where both our designed acoustic patterns and existing MNIST digit patterns are used.

\section{Results}

Artificial van der Waals hybrid synapse. Biological synapses are known to transmit spike signals from the presynaptic terminal to the postsynaptic terminal using neurotransmitters and to adjust their synaptic weights on the basis of the timing of the spike signals $^{32}$. In this study, as shown in Fig. 1a, we implemented an vdW-hybrid synaptic device that successfully mimics the operation of biological synapses and presented excellent synaptic characteristics. This vdW-hybrid synaptic device features two signal paths for potentiation and depression operations, which are formed on $\mathrm{WSe}_{2}$ (for hole transport)/hexagonal-boron nitride ( $h$ $\mathrm{BN})$ and $\mathrm{MoS}_{2}$ (for electron transport) $/ h$-BN heterostructures, respectively. Here, such vdW heterostructures are free from concerns about lattice mismatching owing to the dangling-bondfree surface nature of the vdW materials ${ }^{33-35}$, thereby allowing the formation of interfacial defect-free floating gate structure ${ }^{36,37}$ or the modulation of the number of interfacial traps/dipoles for achieving the synaptic functionalities $12,18,38$. The potentiation and depression channels are tied by two electrodes, which are defined as the presynaptic and postsynaptic terminals, and the two channels have an individual gate electrode functioning as a weight control terminal (WCT). Additional information regarding the vdW-hybrid synaptic device, such as an optical microscopy image, thickness profiles of vdW materials confirmed via atomic force microscopy (AFM), and the Raman spectra of each vdW material are provided in Supplementary Fig. 1. When a voltage spike $\left(V_{\text {pre }}\right)$ is applied to the presynaptic terminal, a postsynaptic current (PSC) appears at the postsynaptic terminal, which is the sum of the PSCs of the potentiation (PSC $C_{\mathrm{P}}$ ) and depression $\left(\mathrm{PSC}_{\mathrm{D}}\right)$ channels $\left(\mathrm{PSC}=\mathrm{PSC}_{\mathrm{P}}+\mathrm{PSC}_{\mathrm{D}}\right)$. This indicates that the conductance of the vdW-hybrid synaptic device $(G)$ is identical to the sum of the conductance values of the potentiation $\left(G_{\mathrm{P}}\right)$ and depression $\left(G_{\mathrm{D}}\right)$ channels $\left(G=G_{\mathrm{P}}+G_{\mathrm{D}}\right)$. Therefore, as shown in Fig. 1b, the synaptic conductance of this device can be potentiated $\left(G \uparrow=G_{\mathrm{P}} \uparrow+G_{\mathrm{D}}\right)$ or depressed $(G \downarrow=$ $\left.G_{\mathrm{P}}+G_{\mathrm{D}} \downarrow\right)$ by applying only a positive voltage spike $\left(+V_{\mathrm{WCT}}\right)$ to the WCT. The conductance of the $\mathrm{WSe}_{2}$ and $\mathrm{MoS}_{2}$ channels is modulated on the basis of the phenomenon that electrons are only trapped in the weight control layer (WCL) formed on $h$-BN under the positive voltage spike condition. This differs from the conventional transistor-type synapse, where both trapping and detrapping of electrons are used for potentiation and depression, which allows highly symmetric operation of the synapse ${ }^{12,39}$. For accurate operation of the vdW-hybrid synaptic device, two different polarity FET devices with a similar current level are needed to be integrated. When voltage spikes with an amplitude of $1 \mathrm{~V}$, a duration of $20 \mathrm{~ms}$, and a frequency of $2 \mathrm{~Hz}$ were applied to the WCT for the potentiation channel four times consecutively, the conductance of the vdW-hybrid synaptic device increased in steps from 159 to $257 \mathrm{nS}$ (potentiation operation). When the same 
a

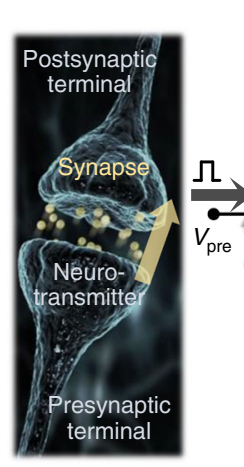

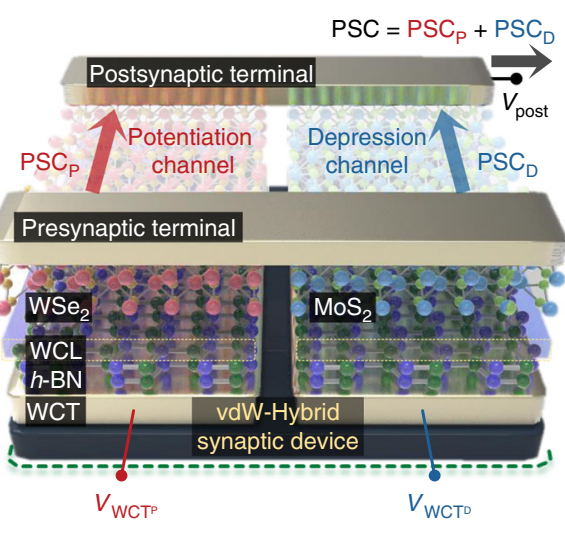

b
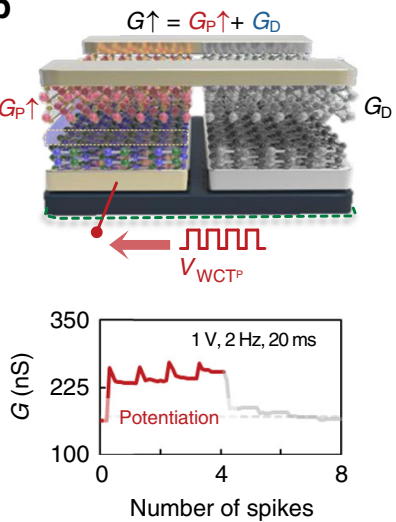
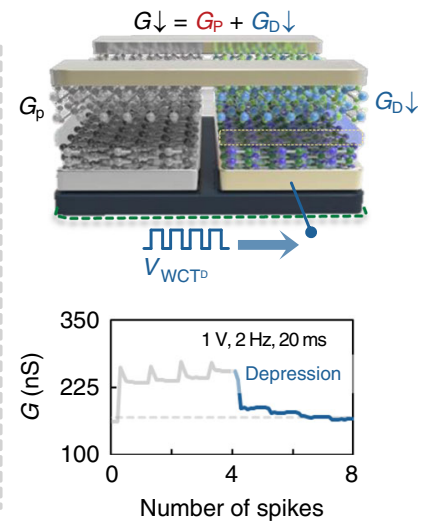

C
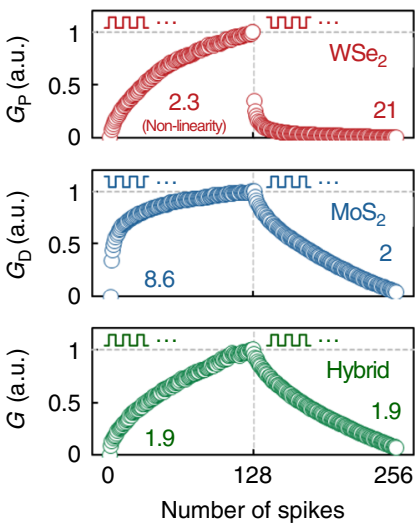

d
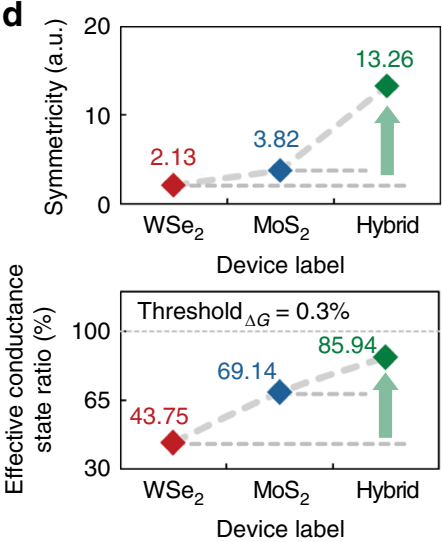

e
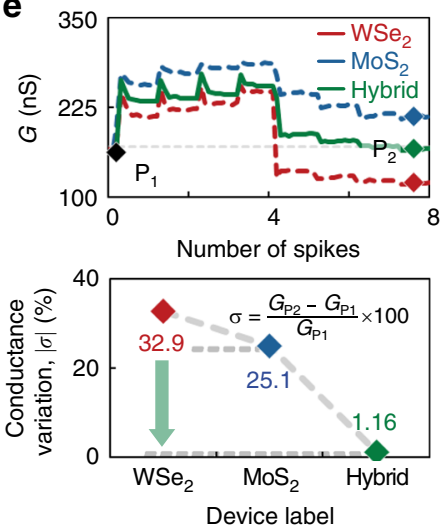

Fig. 1 vdW-hybrid synaptic device with excellent controllability of the conductance. a Functional and structural comparison of the biological synapse with the vdW-hybrid synaptic device. b Demonstration of potentiation and depression operations with four spikes. c Long-term potentiation (LTP) and depression (LTD) curves including extracted nonlinearity values for the control devices (WSe 2 and $\mathrm{MoS}_{2}$ synaptic devices) and the vdW-hybrid synaptic device, where 128 excitatory and inhibitory spikes were applied consecutively to the WCTs. $\mathbf{d}$ Symmetricity and effective conductance-state ratio (threshold ${ }_{\Delta G}=0.3 \%$ ) extracted from the LTP/LTD curves. e Conductance responses when eight spikes were applied in a row (four excitatory and four inhibitory) to the WCTs of the three types of devices, and extracted conductance variations $|\sigma|$.

voltage spikes were applied to the WCT for the depression channel, the conductance decreased in steps to $163 \mathrm{nS}$, which was similar to the initial conductance value (depression operation).

Following the demonstration of the selective controllability of the conductance in the vdW-hybrid synaptic device, we compared its synaptic characteristics with those of a control synaptic device with a $\mathrm{WSe}_{2}$ or $\mathrm{MoS}_{2}$ channel. As shown in Fig. 1c, we confirmed the LTP/LTD characteristics in each synaptic device, where 128 excitatory and inhibitory voltage spikes were applied in a row to the WCTs of the devices. For the $\mathrm{WSe}_{2}$ synaptic device, the conductance increased linearly and decreased nonlinearly (red curve). The conductance change of the $\mathrm{MoS}_{2}$ synaptic device (blue curve) exhibited the opposite behavior. According to these results, the channels composed of WSe $e_{2}$ and $\mathrm{MoS}_{2}$ were specialized for potentiation and depression, respectively. Therefore, in the vdW-hybrid synaptic device that selectively exploits the specialized channels for the LTP/LTD, the conductance states are uniformly distributed (green curve). The measurement setup for the synaptic devices is described in detail in Supplementary Fig. 2. To evaluate the LTP/LTD characteristics quantitatively, we extracted the nonlinearity from the characteristic curves ( $\beta_{\mathrm{P}}$ from the LTP curve and $\beta_{\mathrm{D}}$ from the LTD curve). The nonlinearity values $\left(\beta_{\mathrm{P}} / \beta_{\mathrm{D}}\right)$ of the $\mathrm{WSe}_{2}$ and $\mathrm{MoS}_{2}$ synaptic devices were $2.3 / 21$ and $8.6 / 2$, respectively. For the vdW-hybrid synaptic device, the nonlinearity values were 1.9/1.9. The dynamic ranges of the $\mathrm{WSe}_{2}, \mathrm{MoS}_{2}$, and vdW-hybrid synaptic devices, which were defined as the difference between $G_{\max }$ and
$G_{\min }\left(G_{\max }-G_{\min }\right)$, were 237,210 , and $191 \mathrm{nS}$, respectively. These nonlinearity values were analyzed and compared with the values of previously reported artificial synapses, as shown in Supplementary Fig. 3. We then calculated the symmetricity indicating the degree of symmetry between the LTP and LTD characteristic curves in Fig. 1d (top). Details regarding the calculation are provided in Supplementary Fig. 4. The symmetricity values of the $\mathrm{WSe}_{2}$ and $\mathrm{MoS}_{2}$ synaptic devices were approximately 2.13 and 3.82 , respectively, and the symmetricity was improved to 13.26 for the vdW-hybrid channel. Here, there are flake-to-flake variations in the vdW channels in terms of defect density and doping concentration, which consequently affect the synaptic characteristics including the linearity and symmetricity of the LTP and LTD curves. Detailed analysis regarding with this issue is provided in Supplementary Fig. 5. In addition, the nonlinearity and symmetricity values were investigated in multiple samples to confirm the DDV in the nonlinearity and symmetricity, as shown in Supplementary Fig. 6. Furthermore, we determined and compared the effective conductance-state ratios, as shown in Fig. 1d (bottom), because an insufficient conductance change $(\Delta G)$ in the LTP and LTD curves has no effect on the recognition rate in the HW-NN. The effective conductance-state ratio was defined as the ratio of the number of conductance states in which $\Delta G$ exceeded a certain percentage of $G_{\max } / G_{\min }\left(\right.$ threshold $\left._{\Delta G}\right)$ to the total number of conductance states. When threshold ${ }_{\Delta G}$ was set as $0.3 \%$, the $\mathrm{WSe}_{2}$ and $\mathrm{MoS}_{2}$ synaptic devices exhibited effective conductance-state ratios of $43.75 \%$ and $69.14 \%$, 
respectively, and the vdW-hybrid synaptic device exhibited a relatively high ratio of $85.94 \%$. As shown in Fig. 1e, we applied eight spikes in a row for potentiation and depression (four excitatory spikes and four inhibitory spikes) to the WCTs of the three devices. Consequently, the conductance of the $\mathrm{WSe}_{2}$ and $\mathrm{MoS}_{2}$ devices decreased (conductance variation $\sigma=-32.9 \%$ ) and increased $(\sigma=+25.1 \%)$, respectively, compared with the initial values. For the hybrid synaptic device, a conductance similar to the initial value was observed under the same spike conditions $(\sigma=+1.16 \%)$. Here, the conductance variation $\sigma$ was calculated using the equation shown in Fig. 1e (bottom). Additional results measured under different combinations of eight spikes are provided in Supplementary Fig. 7, (e.g., two excitatory spikes, two inhibitory spikes, two excitatory spikes, and two inhibitory spikes).

Analysis of vdW-hybrid synaptic device. The excellent conductance controllability of the vdW-hybrid synaptic device was due to the electron-trapping phenomenon in the WCL, which was formed by exposing $\mathrm{CF}_{4}$ plasma on top of the $h$-BN (details are presented in the METHODS Section). As shown in Fig. 2a, we examined the WCL via cross-sectional transmission electron microscopy (X-TEM). By performing $\mathrm{CF}_{4}$ plasma treatment with a reactive ion etcher power of $10 \mathrm{~W}$ and a process time of $10 \mathrm{~s}$, the WCL was created at depths of 11.1 and $10.5 \mathrm{~nm}$ from the surface underneath the potentiation and depression channels, respectively. The regions inside the yellow dotted line in Fig. 2a were analyzed via electron energy-loss spectroscopy (EELS) mapping, yielding atomic-composition information for each region. As shown in Fig. $2 \mathrm{~b}$ and $\mathrm{c}$, the WCL regions mainly exhibited signals corresponding to C (green), F (yellow), and B (weak signal, blue). As expected, signals corresponding to $\mathrm{W}$ (bright red), Se (bright green), Mo (purple), and S (cyan) were obtained in the regions of $\mathrm{WSe}_{2}$ and $\mathrm{MoS}_{2}$. The B (blue) and N (red) signals also appeared in the region of $h$-BN. As shown in Supplementary Figs. 8, 9, and 10 , we analyzed the energy distribution of the WCL/h-BN via micro photoluminescence and micro X-ray photoelectron spectroscopy measurements ${ }^{40-42}$. Additionally, we estimated the trap density in the WCL region containing $\mathrm{C}$ and $\mathrm{F}$ atoms, obtaining density values of $5.2 \times 10^{17}$ and $5.8 \times 10^{17} \mathrm{~cm}^{-3}$ for the potentiation and depression channels, respectively. These trap-density values were on the same order as the number of electrons stored in the floating gate of current flash memory cell, as discussed in Supplementary Fig. 11 (http://www.itrs2.net/). As depicted in Fig. 2d, we investigated carrier injection barrier heights and work functions for the $\mathrm{WSe}_{2}$ (potentiation) and $\mathrm{MoS}_{2}$ (depression) channels via the modified Richardson plotting method, based on a thermionic emission-diffusion model and Kelvin probe force microscopy (KPFM) analysis, respectively (see also Supplementary Fig. 12 and Note 8) ${ }^{43,44}$. A hole barrier height of $0.31 \mathrm{eV}$ and work function of 4.86 were estimated for the $\mathrm{WSe}_{2}$ channel, where a platinum contact is formed between the pre/postsynaptic terminals and channel. Meanwhile, an electron barrier height of $0.19 \mathrm{eV}$ and work function of $4.75 \mathrm{eV}$ were obtained for the $\mathrm{MoS}_{2}$ channel, where a titanium contact is formed between the pre/ postsynaptic terminals and channel. Therefore, the $\mathrm{WSe}_{2}$ (potentiation) and $\mathrm{MoS}_{2}$ (depression) channels were confirmed as $\mathrm{p}$ - and n-type channels, respectively. We then investigated the spike responses for the current flow through the potentiation and depression channels in detail. When an excitatory voltage spike was applied to the WCTs for the WSe $\mathrm{W}_{2}$ and $\mathrm{MoS}_{2}$ channels, the PSCs flowing through the potentiation and depression channels increased from 20.2 to $25.6 \mathrm{nA}$ and decreased from 21.1 to $16.1 \mathrm{nA}$ for $10^{4}$ seconds, denoting that the trap states of WCL have high confinement energy (see Fig. 2d). This is because the electrons trapped in the WCL increased and decreased the number of holes and electrons in the $\mathrm{WSe}_{2}$ and $\mathrm{MoS}_{2}$ channels, respectively, decreasing and increasing the width of the tunneling barrier $\left(W_{\mathrm{TN}}\right)$ from the presynaptic terminal $\left(T_{\mathrm{pre}}\right)$ metal to the vdW channels, as shown in Fig. 2d (bottom). When an inhibitory spike was applied, as shown in Supplementary Fig. 13, the PSC decreased and increased in the potentiation and depression channels, respectively. The operating energy for reading and updating a weight were approximately from 0.12 to $0.71 \mathrm{~nJ}$ (for reading energy) and 0.79 (for updating energy in the potentiation channel)/0.93 (for updating energy in the depression channel) $\mathrm{pJ}$, respectively, where a spike with $1 \mathrm{~V}$ of amplitude and $10 \mathrm{~ms}$ of duration was applied ${ }^{45}$. Such dissipated energy per event were determined by $P=I \times V \times t_{\text {duration }}{ }^{12,16,18}$, and relevant details are provided in Supplementary Fig. 14. The PSC responses with respect to the amplitude and duration of the spike and with respect to the conditions of $\mathrm{CF}_{4}$ plasma treatment were also investigated, as shown in Supplementary Fig. 15. As shown in Fig. 2e, when excitatory spikes with a 1-V amplitude, 20-ms duration, and $2-\mathrm{Hz}$ frequency were applied consecutively to the WCTs underneath the $\mathrm{WSe}_{2}$ and $\mathrm{MoS}_{2}$ channels, the conductance linearly increased (Case 1) and decreased (Case 2), respectively. Under the excitatory-spike condition, the energy band of the semiconductor near the WCL was instantly bent downward, generating an electric field $(E)$ that attracted electrons toward the WCL (see Fig. 2f). Simultaneously, the probability of trap states being filled increased because the Fermi level of the semiconductor was close to its conduction band edge. Therefore, the trap sites at the WCL were partially filled with electrons during each excitatory spike, which gradually changed the conductance of the potentiation and depression channels. In contrast, as shown in Fig. $2 \mathrm{~g}$, when the same inhibitory voltage spikes were applied in a row, the conductance nonlinearly decreased and increased at the $\mathrm{WSe}_{2}$ and $\mathrm{MoS}_{2}$ channels, respectively. The energy band of the semiconductor near the WCL was instantly bent upward when the inhibitory spike was applied, generating an electric field for electron detrapping (see Fig. 2h). Additionally, because there were many empty states within the conduction band of the semiconductor, which were well aligned with the filled trap sites in the energy level, most of the trapped electrons were released from the WCL during the initial few inhibitory spikes. Consequently, when the inhibitory spikes were applied in a row after the excitatory spikes, the conductance rapidly changed at the beginning stages and then gradually became saturated. In the proposed hybrid synapse device, the linearity of PSC was determined by (i) the PSC updating origin (electron trapping into the WCL or detrapping from the WCL), and (ii) the polarity of the channel (p- or n-type channel) (see Supplementary Fig. 16). We further investigated the spike response for the synaptic device using an $\mathrm{MoSe}_{2}$ that normally functions as an n-type channel and subsequently confirmed the similar LTP/LTD characteristics of the $\mathrm{MoSe}_{2}$ and $\mathrm{MoS}_{2}$ synaptic devices (see Supplementary Fig. 17). Meanwhile, the nonlinearity for the potentiation and depression channels was investigated with respect to (i) the amplitudes and durations of the spikes, and (ii) the conditions of $\mathrm{CF}_{4}$ plasma treatment, as shown in Supplementary Fig. 18. The information on the spike responses for the potentiation and depression channels without WCL is provided in Supplementary Fig. 19.

Synaptic characteristics of the vdW-hybrid synaptic device with respect to various spike conditions. Next, we examined the CCV and RSD for the LTP/LTD curves and the symmetricity and dynamic range of the vdW-hybrid device under various voltage spike conditions. These indices significantly affect the 
a

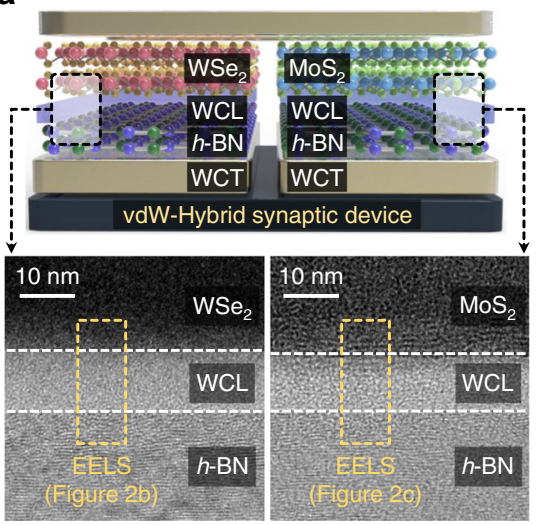

b

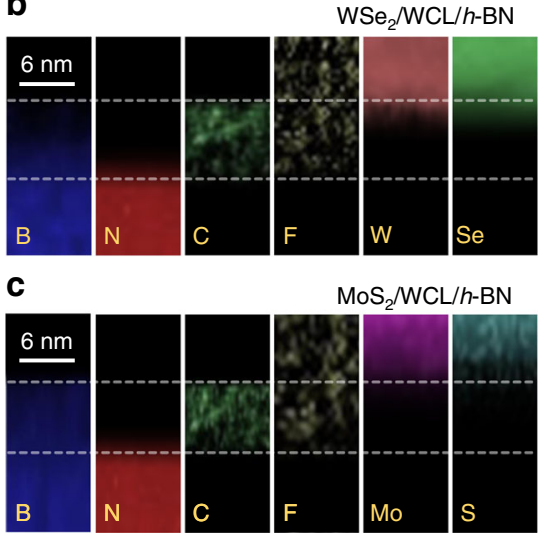

d

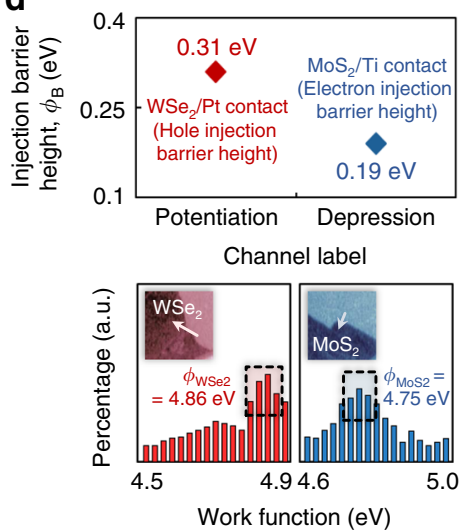

e
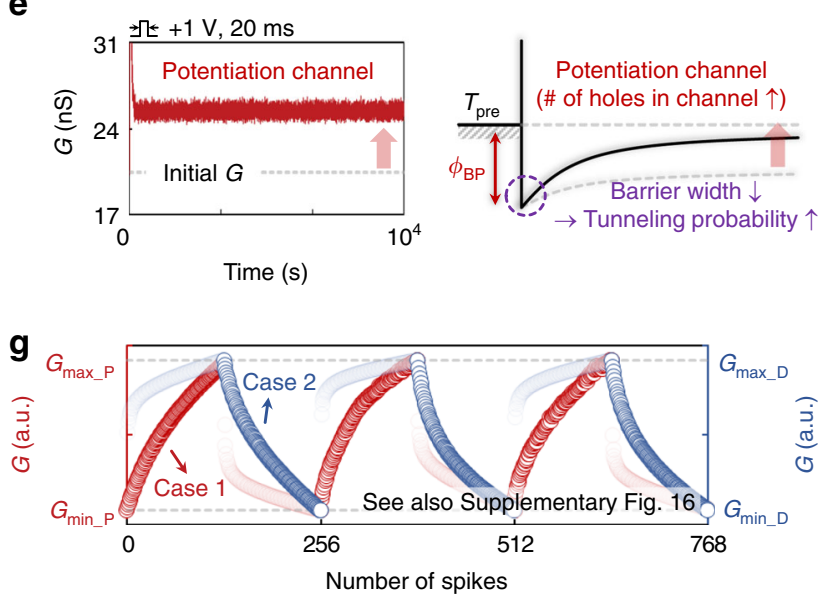

h Electron-trapping into the WCL (for Case 1 and Case 2)

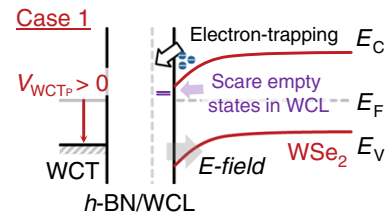

f
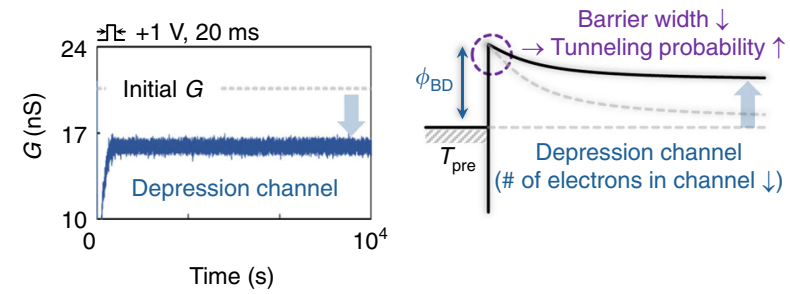

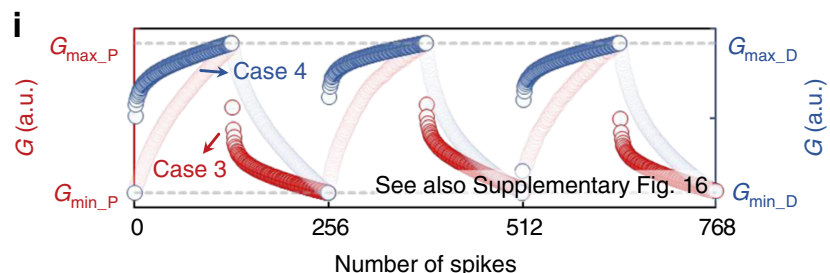

j Electron de-trapping from the $\mathrm{WCL}$ (for Case 3 and Case 4)
Case 3

Fig. 2 Study on the weight control mechanism of the vdW-hybrid synaptic device. a X-TEM images of the WSe $2 / W C L / h-B N(p o t e n t i a t i o n ~ c h a n n e l)$ and $\mathrm{MoS}_{2} / \mathrm{WCL} / h$-BN (depression channel) structures. b, $\mathbf{c}$ EELS mapping images obtained on the cross-sectional regions. $\mathbf{d}$ Extracted injection barrier height and work function for the potentiation and depression channels. e, $\mathbf{f}$ Excitatory (e) and inhibitory (f) PSC in the potentiation and depression channels. Illustration of energy band diagrams when an excitatory spike was applied to the WCTs for the potentiation and depression channels. $\mathbf{g}$ LTP curves through the $\mathrm{WSe}_{2}$ channel (Case 1) and LTD curves through the $\mathrm{MoS}_{2}$ channel (Case 2), where 128 excitatory spikes were applied in a row to the WCTs. h Illustration of energy band diagrams describing phenomena of electron trapping into the WCL. i LTP curves through MoS 2 channel (Case 3) and LTD curves through $\mathrm{WSe}_{2}$ channel (Case 4), where 128 inhibitory spikes were applied in a row to the WCTs. $\mathbf{j}$ Illustration of energy band diagrams describing phenomena of electron detrapping from the WCL.

performance of an $\mathrm{HW}-\mathrm{NN}$ composed of artificial synapses $^{20,23,46}$. Figure 3 a explains the two voltage spike conditions-one for potentiation and the other for depression-where the number, amplitude, duration, and frequency of the pulses were varied. As shown in Fig. 3b, we measured the LTP/LTD characteristics 15 times and then evaluated the CCV and $\mathrm{RSD}^{21,46}$. The CCV was estimated as $<1 \%$ in the LTP/LTD curves, as shown in Supplementary Fig. 20. Here, the nonlinearity ranging from 1.75 to 2.2 for the potentiation channel and from 1.8 to 2.35 for the depression channel was confirmed. For the $\mathrm{RSD}$, which represents the ratio of the standard deviation $(\sigma)$ to the mean $(\mu)$, values of 0.05 and 0.03 were obtained in the LTP and LTD curves, respectively. Also, as shown in Supplementary Fig. 21, we investigated the endurance $\left(>10^{5}\right.$ weight updating, 500 cycles of LTP/LTD) of the vdW-hybrid device. We then extracted the symmetricity and dynamic range from the LTP/LTD curves obtained when 32,64 , and 128 voltage spikes were applied, as shown in Fig. 3c. While the symmetricity was not significantly affected by the number of spikes, the dynamic range increased rapidly as the number of spikes increased (symmetricity/dynamic range: 7.95/96.12 $\mathrm{nS}$ for 32 states, $8.05 / 124.6 \mathrm{nS}$ for 64 states, and $7.61 / 178 \mathrm{nS}$ for 128 states). In addition to the effects of the number of spikes, we investigated the symmetricity and the dynamic range under different spike voltages. As the amplitude of the spikes increased from 1 to $5 \mathrm{~V}$, the symmetricity decreased from 5.11 to 3.39 , and the dynamic range increased from 95 to $326 \mathrm{nS}$ (see Fig. 3d). As the duration of the spikes increased from 10 to $50 \mathrm{~ms}$, the symmetricity decreased from 11.65 to 6.11 , and 
a

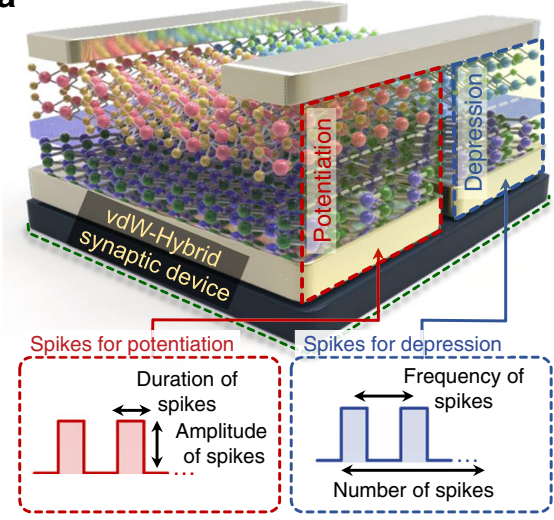

d
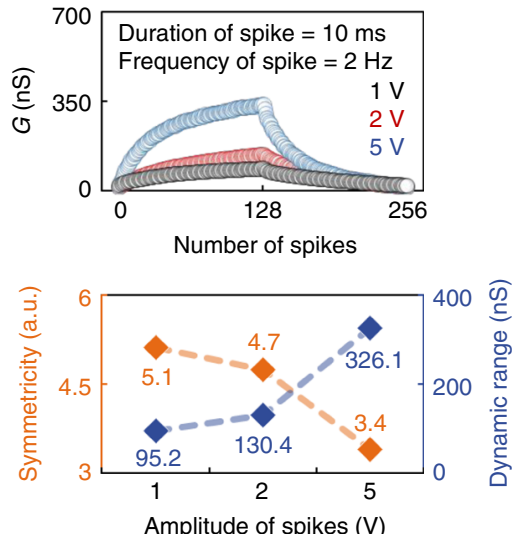

b
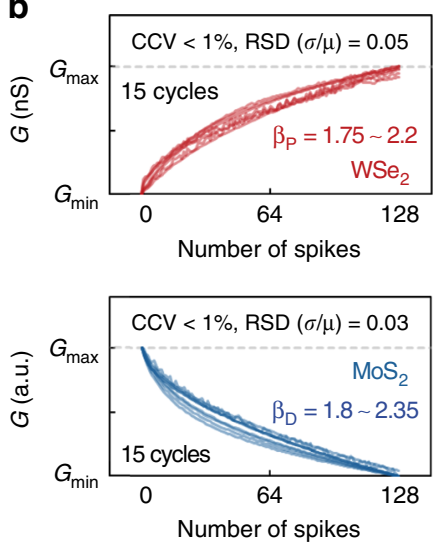

e
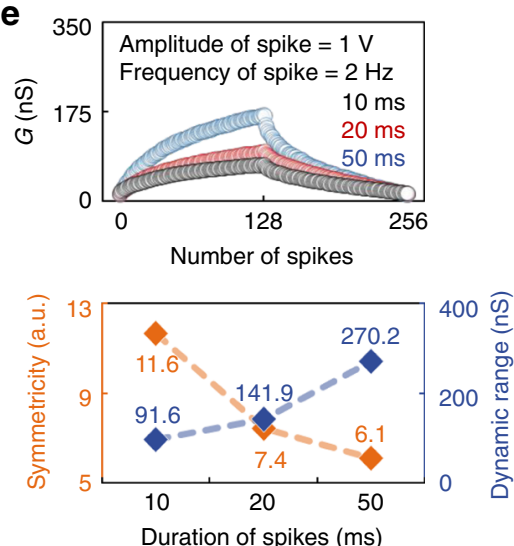
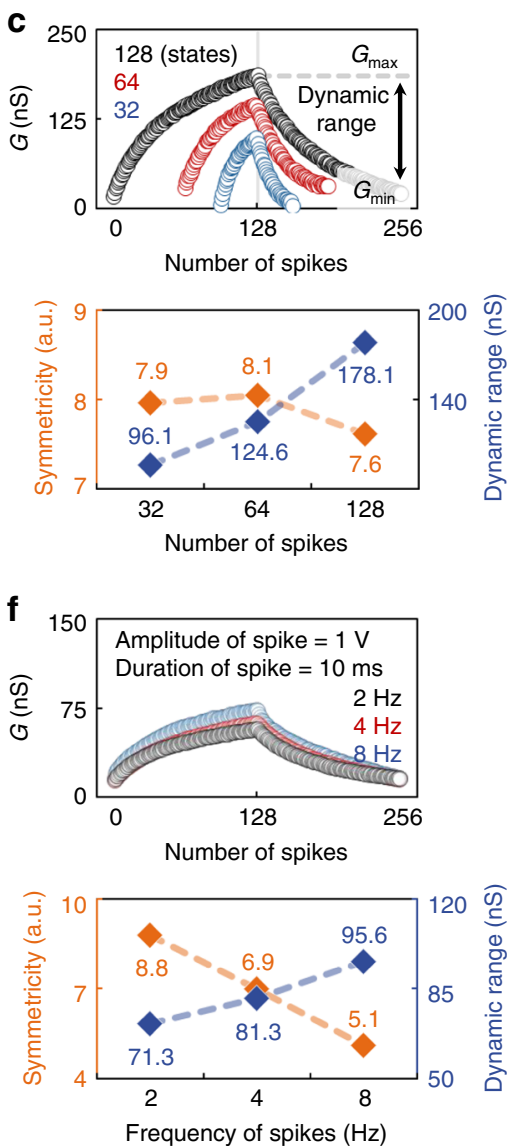

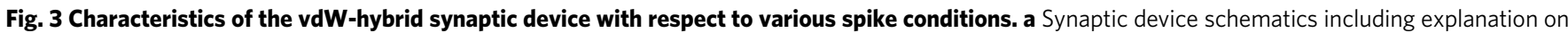
spike condition. b LTP and LTD curves for 15 cycles and evaluated CCV and RSD $(\sigma / \mu)$ values for the potentiation and depression channels. c-f Extracted symmetricity and dynamic range from the LTP/LTD curves measured under various spike conditions, such as the number of spikes (32-128) (c), the amplitude of spikes (1-5 V) (d), the duration of spikes (10-50 ms) (e), and the frequency of spikes (2-8 Hz) (f).

the dynamic range increased from 91 to $270 \mathrm{nS}$ (see Fig. 3e). As the frequency of the spikes increased from 2 to $8 \mathrm{~Hz}$, the symmetricity decreased from 8.79 to 5.11 , and the dynamic range increased from 71 to $95 \mathrm{nS}$ (see Fig. 3f). As the amplitude, duration, and frequency of the spikes increased, the symmetricity was degraded and the dynamic range value increased, indicating that these performance indices had a tradeoff relationship with each other. A detailed analysis of the results for the symmetricity and the dynamic range is provided in Supplementary Fig. 22.

Acoustic pattern recognition task. Finally, we demonstrated the feasibility of the vdW-hybrid synaptic device for an HW-NN via a training and inference simulation. For this simulation task, we defined a method to convert vocal signals into acoustic patterns and then prepared training and inference datasets, as shown in Fig. $4 \mathrm{a}$ and $\mathrm{b}$. The first step was to express vocal signals as a function of time or frequency. We recorded the sound wave of a spoken word ("strawberry") and obtained the sound information as a function of time. The sound amplitude vs. time information was transformed to the frequency domain via a Fourier transform. The second step was sampling the sound signals. In this step, we divided the sound signals into 200 time or frequency points. Finally, in the third step, the discrete signal information was transformed into an acoustic image with a $20 \times 20$ array size, as shown in Fig. 4b. For example, the 109th data point in the sound amplitude vs. time graph and the 61st data point in the sound magnitude vs. frequency graph were transferred into the pixels located at $(6,11)$ and $(14,1)$ in the acoustic pattern, respectively (see dotted red line). Here, each pixel had a grayscale value in the range of $0-255$. Datasets with 3000 training and 400 inference acoustic pattern images were prepared similarly for five distinct words: "apple," "orange," "kiwi," "banana," and "strawberry" (see Fig. 4c). Additional information about the datasets is presented in Supplementary Fig. 23. We also prepared two types of spoken digit datasets consisting of cochleagram patterns or our acoustic patterns, where the Lyon's auditory model was applied to create the cochleagram patterns (see Supplementary Fig. 24) ${ }^{47}$. Then, as shown in Fig. 4d, we theoretically designed a single-layer artificial neural network (ANN) consisting of 400 input neurons, five output neurons, and $400 \times 5$ artificial synapses connecting the neurons. The voltage signals $\left(V_{\mathrm{n}}\right)$ corresponding to each pixel of the acoustic pattern were assumed to be applied to the input neuron layer. They were multiplied by the synapse weight $\left(W_{\mathrm{n}, \mathrm{m}}\right)$ and then summed at the output neurons. Consequently, output currents $\left(I_{\mathrm{m}}=\sum_{n=1}^{400} W_{\mathrm{n}, \mathrm{m}} V_{\mathrm{n}}\right)$ were obtained at the output neuron layer. The synapse weight was defined as the conductance values of the synaptic device $(W=G)$. Next, the output value $\left(f_{\mathrm{m}}\right)$ obtained via the sigmoid activation function $\left(f\left(I_{\mathrm{m}}\right)=\frac{1}{1+e^{-I_{\mathrm{m}}}}\right)$ was compared with each label value $\left(k_{\mathrm{m}}\right)$. Finally, the synapse weights were updated via the backpropagation algorithm (details are presented in the METHODS Section). Figure 4e shows the hardware neural network (HW-NN) comprising the vdW-hybrid synaptic devices, which is applicable to the implementation of the conceptual neural networks for acoustic and MNIST digit pattern 
a

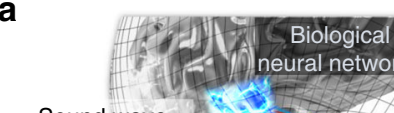

Sound wave

(e.g., Strawberry)

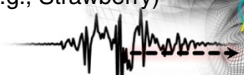

Step \#1:
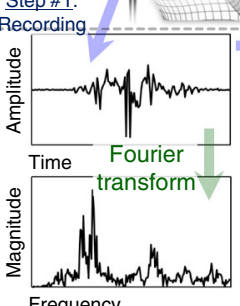

Frequency b

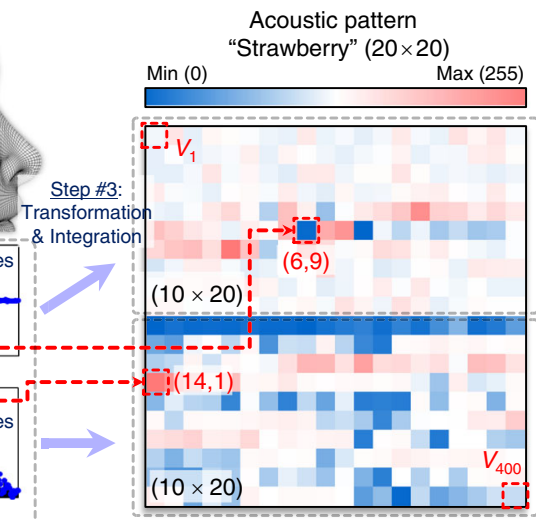

C

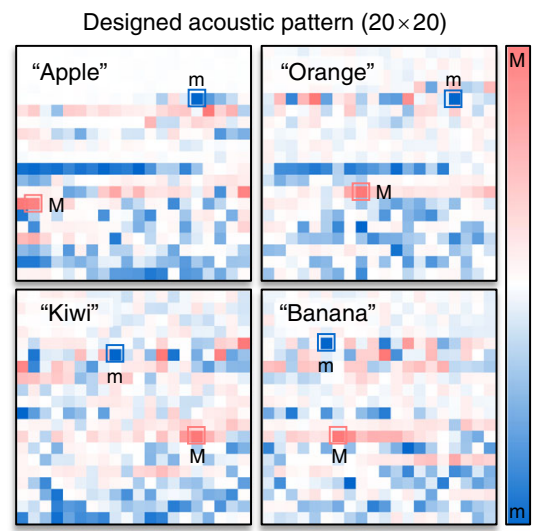

d Artificial neural network (ANN) Input layer Synapse weights Output layer

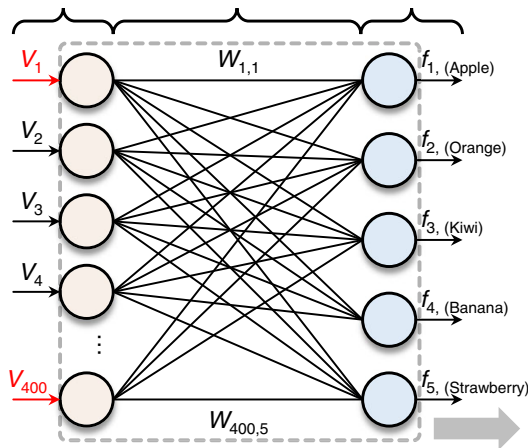

f

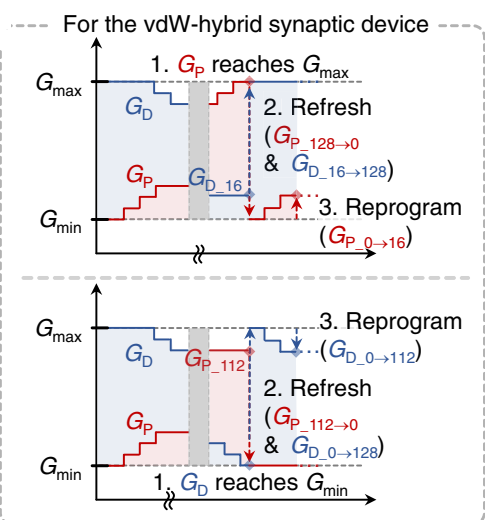

e

.. Hardware neural network (HW-NN) comprising vdW-hybrid synaptic devices -..----

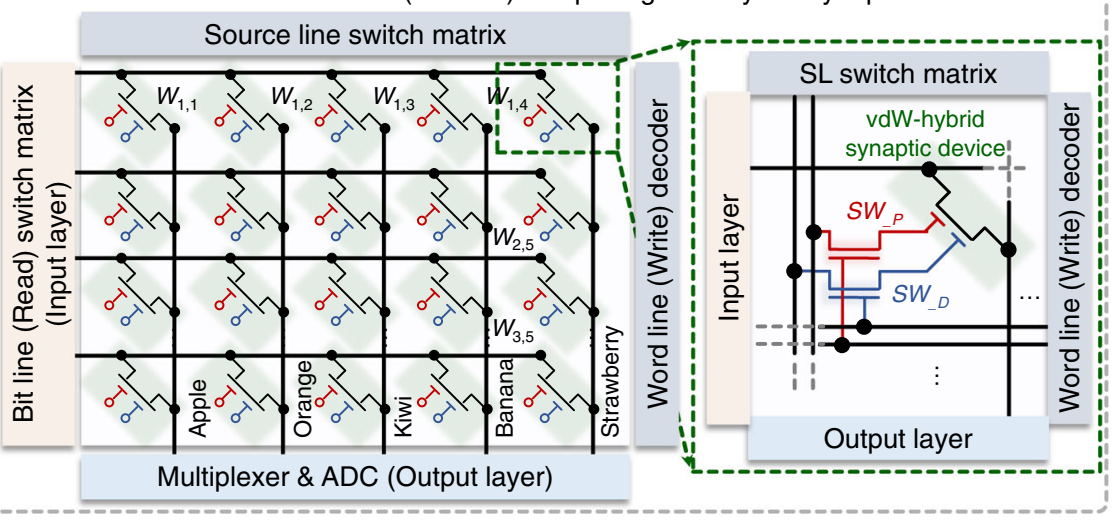

g

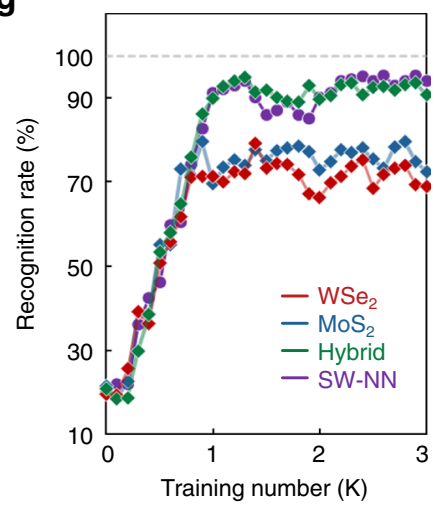

h
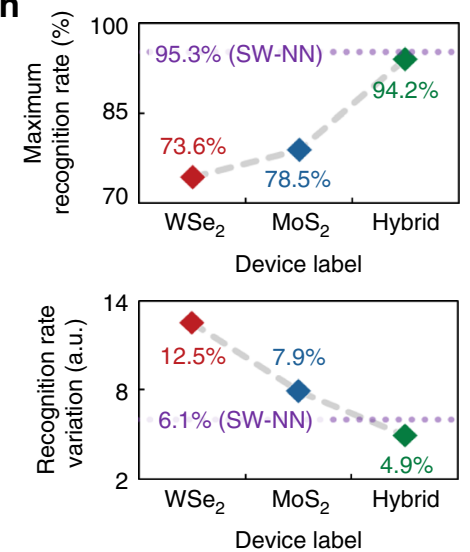

Fig. 4 Acoustic pattern recognition task. a Design procedure of acoustic pattern (from recording, through transforming, to integrating). b Designed $20 \times$ 20 acoustic pattern, where each pixel has a grayscale value in the range between 0 and 255. c Designed acoustic pattern. $\mathbf{d}$ Single-layer artificial neural network (ANN) consisting of input neurons, synapses, and output neurons. e The hardware neural network (HW-NN) comprising the vdW-hybrid synaptic device for the conceptual neural networks. f Weight update method based on operations of "refresh" and "reprogram" for the vdW-hybrid synaptic device. g Acoustic pattern recognition rates based on three ANNs comprising $\mathrm{WSe}_{2}, \mathrm{MoS}_{2}$, or hybrid synaptic devices, and comparison with the rate based on SW-NN. h Extracted maximum recognition rates and corresponding variations.

recognition tasks. Details on this HW-NN are described in Supplementary Fig. 25.

The conductance of the vdW-hybrid synaptic device was updated by only trapping electrons in the WCL, which caused the conductance state to no longer potentiate or depress when $G_{\mathrm{P}}$ or $G_{\mathrm{D}}$ reached $G_{\max }$ or $G_{\min }$. Therefore, for training the ANN composed of the hybrid devices, we employed a conductance updating method based on the operations of "refresh" and "reprogram" for updating $G_{\mathrm{P}}$ and $G_{\mathrm{D}}$. As shown in Fig. $4 \mathrm{f}$ (top), when $G_{\mathrm{P}}$ reached $G_{\max }\left(G_{\mathrm{P}_{-} 128}\right)$, both $G_{\mathrm{P}}$ and $G_{\mathrm{D}}$ were refreshed to $G_{\min }\left(G_{\mathrm{P}_{-} 128 \rightarrow 0}\right)$ and $G_{\max }\left(G_{\mathrm{D}_{1} 16 \rightarrow 128}\right)$. For the operation of "refresh" in terms of implementation in hardware, (i) the peripheral circuits to read the $G_{P}$ and $G_{D}$ separately and (ii) the physical separation of the channels are required simultaneously. Subsequently, $G_{P}$ was reprogrammed to the value of $G_{D}$ before the refreshing step $\left(G_{\mathrm{P}_{-} 0 \rightarrow 16}\right)$, maintaining its conductance value $\left(G=G_{P_{-} 128}+G_{D_{-} 16}=G_{P_{-} 16}+G_{D_{-} 128}\right)$. For the operation of "reprogram" in terms of implementation in hardware, the 
peripheral circuits and memory are required additionally, which will store the conductance value and write it back to the device. Similarly, as shown in Fig. 4 f (bottom), when $G_{\mathrm{D}}$ reached $G_{\text {min }}$ $\left(G_{\mathrm{D} \quad 0}\right), G_{\mathrm{P}}$ and $G_{\mathrm{D}}$ were refreshed, and then $G_{\mathrm{D}}$ was reprogrammed to the value of $G_{\mathrm{P}}$ before the refreshing step $\left(G_{D_{-} \_128 \rightarrow 112}\right)$. We also employed a conductance updating method without the operations of "refresh" and "reprogram" as shown in Supplementary Fig. 26. The training process was conducted for three types of ANNs composed of hybrid (green curve), $\mathrm{WSe}_{2}$ (red curve), and $\mathrm{MoS}_{2}$ (blue curve) devices, and we calculated the recognition rate every 100 training steps, as shown in Fig. $4 \mathrm{~g}$. The same training process was performed with an SW-NN (purple curve), for which the synaptic weights were updated using the Widrow-Hoff learning rule ${ }^{48}$. Also, the recognition rates for the acoustic patterns formed with frequency- and/or timedomain data are provided in Supplementary Fig. 27. After the training and inference tasks, as shown in Fig. 4h, the maximum recognition rates and corresponding variation values, which denote the degree of fluctuating in learning curves, were examined. The maximum recognition rate/variation values were $73.6 \% / 12.5 \%, 78.5 \% / 7.9 \%$, and $94.2 \% / 4.9 \%$ for the $\mathrm{WSe}_{2}, \mathrm{MoS}_{2}$, and vdW-hybrid synaptic devices. The values for the hybrid device were closest to those for the SW-NN (95.3\%/6.1\%). Similar training and inference analyses were performed for (i) various spike conditions (number, amplitude, duration, and frequency of spikes) with the designed acoustic patterns, (ii) different layer numbers of the ANN (single- and multi-layer) using the MNIST datasets, and (iii) the two types of spoken digit datasets consisting of cochleagram patterns or acoustic patterns, as shown in Supplementary Figs. 28, 29, 30, and Supplementary Table 2, respectively ${ }^{21,49}$.

\section{Discussion}

We developed a vdW-hybrid synaptic device featuring linear and symmetric update characteristics by utilizing $\mathrm{WSe}_{2}$ and $\mathrm{MoS}_{2}$ hybrid channels that are specialized for linear conductance potentiation and depression, respectively. Excellent conductance controllability of the vdW-hybrid synapse was achieved by utilizing only electron-trapping phenomenon in the WCL. The vdW-hybrid synaptic device exhibited nonlinearity and symmetricity of $1.9 / 1.9\left(\beta_{\mathrm{P}} / \beta_{\mathrm{D}}\right)$ and 13.26 , respectively, an effective conductance-state ratio of $85.94 \%$ for threshold ${ }_{\Delta G}=0.3 \%$, a very small variation $(\sim 1 \%)$ after state changes by excitatory and inhibitory spikes, a CCV of $<1 \%$, and an RSD of $0.05 / 0.03$ (weight potentiation/depression). Such synaptic characteristics are highlighted in Supplementary Table 1, where our synaptic device is investigated and compared with other devices reported heretofore. Through in-depth analysis and characterization of the vdW-hybrid synaptic device, we demonstrated the feasibility of the device for an HW-NN. It exhibited high recognition rates close to those for an SW-NN via training and inference simulation, in which our designed acoustic patterns were employed. Using this hybrid synaptic device, we achieved recognition of $93.8 \%$ for an acoustic pattern recognition task, which was close to that for the SW-NN (95.3\%). This work indicates the potential for building HW-NNs for highly accurate brain-inspired computing.

\section{Methods}

Fabrication of the synaptic devices. The individual electrodes for the WCT with a width of $20 \mu \mathrm{m}$ were patterned on a 90 -nm-thick $\mathrm{SiO}_{2}$ oxide layer on a heavily Bdoped Si substrate using an optical lithography process, followed by the deposition of 10-nm-thick Ti and 30-nm-thick Au using an electron-beam evaporator. $h$-BN flakes were mechanically transferred onto the WCTs via a residue-free transfer method based on adhesion energy engineering ${ }^{12}$. Then, $\mathrm{CF}_{4}$ plasma treatment was conducted on the $h$-BN flakes using a plasma machine (Miniplasma Cube, PLASMART). For stabilizing the chamber conditions, $\mathrm{CF}_{4}$ gas flowed for $1 \mathrm{~min}$ before the $\mathrm{CF}_{4}$ plasma treatment. The $\mathrm{CF}_{4}$ plasma treatment conditions were as follows: reactive ion etcher powers of 5,10 , and $20 \mathrm{~W}$; a plasma pressure of $500 \mathrm{mTorr} ; \mathrm{CF}_{4}$ flow rate of $5 \mathrm{sccm}$; and treatment times of 10, 20, and $90 \mathrm{~s}$. The WSe $e_{2}$ and $\mathrm{MoS}_{2}$ flakes were then transferred onto the WCL $/ h$-BN via the same transfer method. The postsynaptic and presynaptic electrodes (distance between the two electrodes and width of the electrodes were $5 \mu \mathrm{m}$ ) were patterned on the WSe $2 / \mathrm{WCL} / h-\mathrm{BN}$ (potentiation channel) and $\mathrm{MoS}_{2} / \mathrm{WCL} / h$-BN (depression channel) structure, followed by 10 -nm-thick Pt contact for the potentiation channel and Ti contact for the depression channel and 50-nm-thick Au pad deposition.

Characterization of the synaptic devices. For structural and elemental analyses of the $\mathrm{WSe}_{2} / \mathrm{WCL} / h$-BN and $\mathrm{MoS}_{2} / \mathrm{WCL} / h$-BN regions, X-TEM (JEM ARM $200 \mathrm{~F}$ ) and EELS (GIF Quantum ER, $200 \mathrm{keV}$ ) measurements were performed. Raman analysis was performed at various positions on the $\mathrm{WSe}_{2} / \mathrm{WCL} / h$ $\mathrm{BN}$ and $\mathrm{MoS}_{2} / \mathrm{WCL} / h$-BN samples using a WITec micro-Raman spectrometer system with a frequency-doubled Nd-doped yttrium aluminum garnet $(\mathrm{Nd}-$ YAG) laser beam (532-nm laser excitation). AFM was performed using an NX10 system (Park Systems Corp.). Electrical measurements of the synaptic devices were performed using an HP-4155A semiconductor parameter analyzer connected to a voltage spike generator (Keysight, 33500B). The aforementioned measurement setup for the synaptic devices is described in detail in Supplementary Fig. 2.

Weight update for synaptic devices. Currents at output neurons were transformed by a sigmoid activation function, resulting in output neuron signals $(f)$. Based on the delta value $(\delta)$, which is difference between the output neuron signals and the label values $(k)$ for input patterns $(\delta=k-f)$, the synaptic weight was determined to be potentiated or depressed. If $\delta>0$ (potentiation phase), then $G$ is increased. In the depression phase $(\delta<0), G$ is decreased. These conductance changes $(\Delta G)$ were determined by the following equations:

$$
\begin{gathered}
G_{\mathrm{n}+1}=G_{\mathrm{n}}+\Delta G_{\mathrm{P}}=G_{\mathrm{n}}+\alpha_{\mathrm{P}} e^{-\beta_{\mathrm{P}} \frac{G_{\mathrm{n}}-G_{\min }}{G_{\max }-G_{\min }}}(\Delta G>0, G \uparrow), \\
G_{\mathrm{n}+1}=G_{\mathrm{n}}+\Delta G_{\mathrm{D}}=G_{\mathrm{n}}-\alpha_{\mathrm{D}} e^{-\beta_{\mathrm{D}} G_{\max }-G_{\min }}(\Delta G<0, G \downarrow) .
\end{gathered}
$$

In these equations, $G_{\mathrm{n}+1}$ and $G_{\mathrm{n}}$ denote the synaptic conductance when the $\mathrm{n}+1$ th and nth pulses are applied, and parameters $\alpha$ and $\beta$ are the conductance change amount and the nonlinearity, respectively. Fitting results are provided in Supplementary Table 3 . The above pattern recognition processing was implemented with MATLAB.

\section{Data availability}

The data that support the findings of this study are available from the corresponding author upon request.

\section{Code availability}

Code from this study (MATLAB scripts) is available from the corresponding author upon request.

Received: 23 November 2019; Accepted: 20 July 2020; Published online: 07 August 2020

\section{References}

1. Backus, J. Can programming be liberated from the von Neumann style?: a functional style and its algebra of programs. Commun. ACM 21, 613-641 (1978).

2. Mead, C. Neuromorphic electronic systems. Proc. IEEE 78, 1629-1636 (1990)

3. Churchland, P. S. \& Sejnowski, T. J. The Computational Brain (MIT Press, Cambridge, 1992).

4. Merolla, P. A. et al. A million spiking-neuron integrated circuit with a scalable communication network and interface. Science 345, 668-673 (2014).

5. Burr, G. W. et al. Experimental demonstration and tolerancing of a large-scale neural network (165 000 synapses) using phase-change memory as the synaptic weight element. IEEE Trans. Electron Devices 62, 3498-3507 (2015).

6. Ohno, T. et al. Short-term plasticity and long-term potentiation mimicked in single inorganic synapses. Nat. Mater. 10, 591-595 (2011).

7. Shi, Y. et al. Electronic synapses made of layered two-dimensional materials. Nat. Elect. 1, 458-465 (2018).

8. Yu, S. et al. An electronic synapse device based on metal oxide resistive switching memory for neuromorphic computation. IEEE Trans. Electron Devices 58, 2729-2737 (2011).

9. Prezioso, M. et al. Training and operation of an integrated neuromorphic network based on metal-oxide memristors. Nature 521, 61-64 (2015). 
10. Zhang, W. et al. Designing crystallization in phase-change materials for universal memory and neuro-inspired computing. Nat. Rev. Mater. 4, 150-168 (2019).

11. Wong, H.-S. P. et al. Phase change memory. Proc. IEEE 98, 2201-2227 (2010).

12. Seo, S. et al. Artificial optic-neural synapse for colored and color-mixed pattern recognition. Nat. Commun. 9, 5106 (2018).

13. Shi, J. et al. A correlated nickelate synaptic transistor. Nat. Commun. 4, 2676 (2013).

14. Kim, M.-K. \& Lee, J.-S. Ferroelectric analog synaptic transistors. Nano Lett. 19, 2044-2050 (2019).

15. Wang, H. et al. A ferroelectric/electrochemical modulated organic synapse for ultraflexible, artificial visual-perception system. Adv. Mater. 30, e1803961 (2018).

16. van de Burgt, Y. et al. A non-volatile organic electrochemical device as a lowvoltage artificial synapse for neuromorphic computing. Nat. Mater. 16, 414-418 (2017).

17. Qian, C. et al. Artificial synapses based on in-plane gate organic electrochemical transistors. ACS Appl. Mater. Interface 8, 26169-26175 (2016).

18. Seo, S. et al. Recent progress in artificial synapses based on two-dimensional van der Waals materials for brain-inspired computing. ACS Appl. Electron Mater. 2, 371-388 (2020).

19. Kang, D.-H. et al. A neuromorphic device implemented on a Salmon-DNA electrolyte and its application to artificial neural networks. Adv. Sci. 6, 1901265 (2019).

20. Sun, J. et al. Optoelectronic synapse based on IGZO-Alkylated graphene oxide hybrid structure. Adv. Funct. Mater. 28, 1804397 (2018).

21. Chen, P.-Y. et al. NeuroSim: a circuit-level macro model for benchmarking neuro-inspired architectures in online learning. IEEE Trans. Comput. Aid. Design Integ. Circuits Syst. 37, 3067-3080 (2018).

22. Lim, S. et al. Adaptive learning rule for hardware-based deep neural networks using electronic synapse devices. Neural Comput. Appl. 31, 8101-8116 (2019).

23. Yu, S. Neuro-inspired computing with emerging nonvolatile memorys. Proc. IEEE 106, 260-285 (2018).

24. Ambrogio, S. et al. Equivalent-accuracy accelerated neural-network training using analogue memory. Nature 558, 60-67 (2018).

25. Burr, G. W. et al. Neuromorphic computing using non-volatile memory. $A d v$. Phys. X 2, 89-124 (2017)

26. Woo, J. et al. Improved synaptic behavior under identical pulses using $\mathrm{AlO}_{\mathrm{X}} /$ $\mathrm{HfO}_{2}$ bilayer RRAM array for neuromorphic systems. IEEE Electron Device Lett. 37, 994-997 (2016).

27. Park, S. et al. Neuromorphic speech systems using advanced ReRAM-based synapse. IEEE Int. Electron Devices Meeting (IEDM). https://ieeexplore.ieee. org/document/6724692 (2013).

28. Jerry, M. et al. Ferroelectric FET analog synapse for acceleration of deep neural network training. IEEE Int. Electron Devices Meeting (IEDM). https:// ieeexplore.ieee.org/document/8268338 (2017).

29. Fuller, E. J. et al. Parallel programming of an ionic floating-gate memory array for scalable neuromorphic computing. Science 364, 570-574 (2019).

30. Kim, S. et al. Analog CMOS-based resistive processing unit for deep neural network training. IEEE 60th MWSCAS. https://ieeexplore.ieee.org/document/ 8052950 (2017).

31. Sun, X. et al. Exploiting hybrid precision for training and inference: A $2 \mathrm{~T}$ 1 FeFET based analog synaptic weight cell. IEEE Int. Electron Devices Meeting (IEDM). https://ieeexplore.iee.org/document/8614611 (2018).

32. Foster, M. \& Sherrington, C. S. Textbook of Physiology. (Macmillan, 1897).

33. Geim, A. K. \& Grigorieva, I. V. Van der Waals heterostructures. Nature 449, 419-425 (2013).

34. Novoselov, K. S. et al. 2D materials and van der Waals heterostructures. Science 353, aac9439 (2016).

35. Shim, J. et al. Electronic and optoelectronic devices based on two-dimensional materials: from fabrication to application. Adv. Elect. Mater. 3, 1600364 (2017).

36. Paul, T. et al. A high-performance $\mathrm{MoS}_{2}$ synaptic device with floating gate engineering for neuromorphic computing. 2D Mater. 6, 045008 (2019).

37. Choi, M. S. et al. Controlled charge trapping by molybdenum disulphide and graphene in ultrathin heterostructured memory devices. Nat. Commun. 4, 1624 (2013).

38. Liu, B. et al. A Fluorographene-based synaptic transistor. Adv. Mater. Tech. 4, 1900422 (2019).

39. Arnold, A. J. et al. Mimicking neurotransmitter release in chemical synapses via hysteresis engineering in $\mathrm{MoS}_{2}$ transistors. ACS Nano 11, 3110-3118 (2017).
40. Tran, T. T. et al. Quantum emission from hexagonal boron nitride monolayers. Nat. Nanotech. 11, 37-41 (2016)

41. Luo, X. et al. Reversible photo-induced doping in $\mathrm{WSe}_{2}$ field effect transistors. Nanoscale 11, 7358-7363 (2019).

42. Museur, L. et al. Defect-related photoluminescence of hexagonal boron nitride. Phys. Rev. B. 78, 155204 (2008).

43. Hastas, N. A. et al. Electrical transport and low frequency noise characteristics of $\mathrm{Au} / \mathrm{n}-\mathrm{GaAs}$ Schottky diodes containing InAs quantum dots. Semicon. Sci. Tech. 19, 461-467 (2004)

44. Lin, Y.-F. et al. Barrier inhomogeneities at vertically stacked graphene-based heterostructures. Nanoscale 6, 795-799 (2014).

45. Yang, C.-S. et al. All-solide-state synaptic transistor with ultralow conductance for neuromorphic computing. Adv. Funct. Mater. 27, 1804170 (2018).

46. Kim, S. et al. Impact of synaptic device variations on pattern recognition accuracy in a hardware neural network. Sci. Rep. 8, 2638 (2018).

47. Lyon, R. F. et al. A computational model of filtering, detection, and compression in the cochlea. Proceed of IEEE-ICASSP-82. 1282-1285 (1982).

48. Widrow, B. et al. Stationary and nonstationary learning characteristics of the LMS adaptive filters. Proc. IEEE 64, 1151-1162 (1976)

49. LeCun, Y. et al. Deep learning. Nature 521, 436-444 (2015).

\section{Acknowledgements}

This research was supported by the Basic Science Research Program, Basic Research Lab Program, and Nano-Material Technology Development Program through National Research Foundation of Korea (NRF) grants funded by the Korean government (MSIP) (2020R1A4A2002806, 2019M3F3A1A01074451, 2018R1A2A2A05020475, and 2016M3A7B4910426)

\section{Author contributions}

S.S. and P.J.-H. designed the experiments and analyzed the data. K.B.-S. and R.H.-J. contributed to the device fabrication. L.J.-J., K.H., and O.S. performed the X-TEM, EELS, XPS, and PL measurements. K.S., S.J., and H.K. performed the pattern recognition simulation. P.J.-H. supervised the research. All authors have discussed the results and commented on the manuscript.

\section{Competing interests}

The authors declare no competing interests.

\section{Additional information}

Supplementary information is available for this paper at https://doi.org/10.1038/s41467 020-17849-3.

Correspondence and requests for materials should be addressed to J.-H.P.

Peer review information Nature Communications thanks Christopher H Bennett and the other, anonymous, reviewers for their contribution to the peer review of this work

Reprints and permission information is available at http://www.nature.com/reprints

Publisher's note Springer Nature remains neutral with regard to jurisdictional claims in published maps and institutional affiliations.

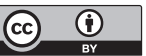

Open Access This article is licensed under a Creative Commons Attribution 4.0 International License, which permits use, sharing, adaptation, distribution and reproduction in any medium or format, as long as you give appropriate credit to the original author(s) and the source, provide a link to the Creative Commons license, and indicate if changes were made. The images or other third party material in this article are included in the article's Creative Commons license, unles indicated otherwise in a credit line to the material. If material is not included in the article's Creative Commons license and your intended use is not permitted by statutory regulation or exceeds the permitted use, you will need to obtain permission directly from the copyright holder. To view a copy of this license, visit http://creativecommons.org/ licenses/by/4.0/

(c) The Author(s) 2020 